\title{
GENERALIZED SUBDIFFERENTIALS OF THE SIGN CHANGE COUNTING FUNCTION
}

\author{
DOMINIQUE FORTIN AND IDER TSEVEENDORJ
}

\begin{abstract}
The counting function on binary values is extended to the signed case in order to count the number of transitions between contiguous locations. A generalized subdifferential for this sign change counting function is given where classical subdifferentials remain intractable. An attempt to prove global optimality at some point, for the 4-dimensional first non trivial example, is made by using a sufficient condition specially tailored among all the cases for this subdifferential.
\end{abstract}

\section{INTRODUCTION}

Two main lines of research are currently challenging in the non smooth optimization field: the rank function [5, for its prominent usage in many real life applications and the extended subdifferentials [12, for tailoring new search techniques for improving local solutions. The rank function is intimately related with both the singular values and the eigenvalues in case of symmetric matrices [6]; the latter case itself, greatly impacts the decomposition of a function into a difference of convex functions (DC decomposition). However, the 0 spectrum remains mixed with either the strictly positive or the strictly negative eigenvalues and most of its significance is usually lost during the standard treatment which linearizes the difficult part of the DC decomposition. Motivated by this drawback, we are aiming at a three way decomposition of the spectrum instead. On the other hand, more subdifferentials have been proposed to be able to prove either necessary and sufficient or sufficient optimality conditions for some classes of non smooth functions to be minimized. In spite of this stream, we are motivated to design rather higher order than $\epsilon$ approximated subdifferentials.

In this article we address both aspects by introducing the sign change counting function, a natural extension to the sign counting function at the heart of the rank function.

In section 2, we remind the notations for the usual subdifferentials with their inclusion chain; in section 3 we revisit the sign counting function results 8 by giving them a concise proof along with bounds, in order to facilitate the study of the sign change counting function. The Q-subdifferential is introduced in section 4 where its relationship with usual and extended subdifferentials is proven. A sufficient condition is derived in a particular case and its effectiveness is shown by proving global optimality of a 1-dimensional function borrowed from the litterature in this stream. Finally, a Q-subdifferential is given for the sign change counting function in section 5 and an attempt to prove the sufficient condition above is made on the 4-dimensional first non trivial example.

Key words and phrases. subdifferential, sign counting. 


\section{Notations}

Given an extended real valued function $f: \mathbb{R}^{n} \rightarrow \mathbb{R} \cup\{\infty\}$, we use standard notations for domain, graph, epigraph:

$$
\begin{aligned}
\operatorname{dom}(f) & =\left\{x \in \mathbb{R}^{n} \mid f(x)<\infty\right\} \\
\operatorname{graph}(f) & =\left\{(x, f(x)) \in \mathbb{R}^{n} \times \mathbb{R} \mid x \in \operatorname{dom}(f)\right\} \\
\operatorname{epi}(f) & =\left\{(x, \alpha) \in \mathbb{R}^{n} \times \mathbb{R} \mid f(x) \leq \alpha\right\}
\end{aligned}
$$

and the orthogonal, ball sets are denoted by

$$
\begin{aligned}
& X^{\perp}(x)=\left\{y \in \mathbb{R}^{n} \mid\langle y, x\rangle=0\right\} \\
& B(x, \varrho)=\left\{y \in \mathbb{R}^{n} \mid\|y-x\| \leq \varrho\right\} .
\end{aligned}
$$

It is assumed that $f$ is lower semicontinuous, equivalently epi $(f)$ is a closed subset of $\mathbb{R}^{n} \times \mathbb{R}$. The closure and convex hull operators are denoted by $\mathrm{cl}$ and conv respectively and a function $\mathbb{R} \rightarrow \mathbb{R}$ is implictly extended to a map $\mathbb{R}^{n} \rightarrow \mathbb{R}^{n}$ by applying it componentwise.

When $f$ is differentiable its gradient is denoted by $\nabla f(x)$, otherwise $x^{\star}$ stands for the subgradient of $f$ at $x$ and various the well known subdifferentials are defined respectively in the following ways

$$
\begin{aligned}
& x^{\star} \in \partial^{\mathrm{P}} f(x) \Longleftrightarrow \Longleftrightarrow \\
& \exists \sigma \in \mathbb{R}_{+}, \text {s.t. } f(x) \leq f(y)-\left\langle x^{\star}, y-x\right\rangle+\sigma\|y-x\|^{2} \text { for all } y \in B(x, \varrho), \\
& x^{\star} \in \partial^{\mathrm{V}} f(x) \Longleftrightarrow \Longleftrightarrow C^{1}\left(\mathbb{R}^{n} \rightarrow \mathbb{R}\right), \nabla g(x)=x^{\star} \text { s.t. } f(x) \leq f(y)-g(y)+g(x) \text { for all } y \in B(x, \varrho), \\
& x^{\star} \in \partial^{\mathrm{F}} f(x) \Longleftrightarrow \liminf _{y \rightarrow x, y \neq x} \frac{f(y)-f(x)-\left\langle x^{\star}, y-x\right\rangle}{\|y-x\|} \geq 0, \\
& x^{\star} \in \partial^{\mathrm{L}} f(x) \Longleftrightarrow \exists x_{n}, x_{n}^{\star} \in \partial^{\mathrm{F}} f(x) \text { s.t. }\left\{\begin{array}{l}
\lim _{n \rightarrow \infty} x_{n}=x \\
\lim _{n \rightarrow \infty} f\left(x_{n}\right)=f(x) \\
\lim _{n \rightarrow \infty} x_{n}^{\star}=x^{\star}
\end{array}\right. \\
& x^{\star} \in \partial^{\mathrm{L}} f(x) \Longleftrightarrow \exists x_{n}, x_{n}^{\star} \in \partial^{\mathrm{F}} f(x), \exists t_{n} \rightarrow 0^{+} \text {s.t. }\left\{\begin{array}{l}
\lim _{n \rightarrow \infty} x_{n}=x \\
\lim _{n \rightarrow \infty} f\left(x_{n}\right)=f(x) \\
\lim _{n \rightarrow \infty} x_{n}^{\star}=\infty \\
\lim _{n \rightarrow \infty} t_{n} x_{n}^{\star}=x^{\star}
\end{array}\right. \\
& x^{\star} \in \partial^{\mathrm{C}} f(x) \Longleftrightarrow x^{\star} \in \operatorname{cl} \operatorname{conv}\left(\partial^{\mathrm{L}} f(x)+\partial^{\mathrm{L}} f(x)\right), x \in \operatorname{dom}(f) .
\end{aligned}
$$

Remark 1. Frechet subdifferential appears as a second order approximation of a regular differential, since $\frac{f(y)-f(x)}{\|y-x\|} \geq\left\langle x^{\star}, \frac{y-x}{\|y-x\|}\right\rangle$ in the vicinity of $x$.

For finite dimensional cases, the following inclusion chain is known

$$
\partial^{\mathrm{P}} f(x) \subset \partial^{\mathrm{V}} f(x)=\partial^{\mathrm{F}} f(x) \subset \partial^{\mathrm{L}} f(x) \subset \partial^{\mathrm{C}} f(x) .
$$




\section{The SIGN COUNTING FUnCtion}

The function $\theta$ counting the number of components different from 0 arises in many applications and its subdifferentials have been studied recently in [8]; in this section, we revisit the proofs in a concise way. We recall that the sign function (or signum function) of a real number $\alpha$ is defined as follows:

$$
\operatorname{sgn}(\alpha)=1 \begin{cases}-1, & \text { if } \alpha<0 \\ 0, & \text { if } \alpha=0 \\ 1, & \text { if } \alpha<0\end{cases}
$$

Let active index set and other related sets useful for defining them, be

$$
\begin{aligned}
I^{\|}(x) & =\left\{i \mid \operatorname{sgn}\left(x_{i}\right)=0\right\}, \\
I^{\perp}(x) & =\left\{i \mid i \notin I^{\|}(x)\right\}, \\
X_{I^{\|}}(x) & =\left\{y \in \mathbb{R}^{n} \mid \operatorname{sgn}\left(y_{i}\right)=0 \text { for all } i \in I_{I^{\|}}(x)\right\}, \\
X_{I^{\perp}}(x) & =\left\{y \in \mathbb{R}^{n} \mid \operatorname{sgn}\left(y_{i}\right)=0 \text { for all } i \in I_{I^{\perp}}(x)\right\}, \\
\theta(x) & =\left|I^{\perp}(x)\right|=\sum\left|\operatorname{sgn}\left(x_{i}\right)\right| .
\end{aligned}
$$

Theorem $1([8])$.

$$
\partial^{P} \theta(x)=\partial^{F} \theta(x)=X_{I^{\perp}}(x)
$$

Proof. First observe that $X_{I^{\perp}}(0)=\mathbb{R}^{n} \supset \partial^{\mathrm{F}} \theta(0)$.

$\partial^{\mathrm{F}} \theta(x) \subset X_{I^{\perp}}(x)$ : Any $x$ is a local minimum for $\theta$, i.e. there exists $\varrho$ such that $\theta(x) \leq \theta(y)$ for all $y \in B(x, \varrho)$. We consider $x \neq 0, \theta(y)=\theta(x) \Longleftrightarrow y \in X(x)$. For $y \in X(x) \backslash 0$, there exists $\epsilon>0$ such that for all $0 \leq \tau \leq \epsilon, x+$ $\tau y \in B(x, \varrho)$. Therefore both $\theta(x+\tau y)=\theta(x)$ and $x^{\star} \in \partial^{\mathrm{F}} \theta(x)$ imply $\liminf _{\tau \rightarrow 0} \frac{-\tau\left\langle x^{\star}, y\right\rangle}{\|y\|} \geq 0$. Suppose $\left\langle x^{\star}, y\right\rangle<0$ and consider $\bar{y}=-y$, then $\bar{y} \in X(x)$ and $\left\langle x^{\star}, \bar{y}\right\rangle>0$ a contradiction; therefore $\left\langle x^{\star}, y\right\rangle=0$. Now, suppose $x^{\star} \in X^{\perp}(x) \backslash X_{I^{\perp}}(x)$ then there are at least 2 indices $i, j$ such that $x_{i}^{\star} y_{i}+x_{j}^{\star} y_{j}=0$ for $y \in X(x)$ but then let $\bar{y}=y$ except for index $i$ whose value is $\bar{y}_{i}=-y_{i}$; it leads to $x_{i}^{\star} \bar{y}_{i}+x_{j}^{\star} y_{j}=-2 x_{i}^{\star} y_{i} \neq 0$, a contradiction too.

$X_{I^{\perp}}(x) \subset \partial^{\mathrm{P}} \theta(x): \quad$ By lower semicontinuity, certainly $0 \in \partial^{\mathrm{P}} \theta(x)$, so we may assume $x^{\star} \neq 0$. For $y \in B(x, \xi) \subset B(x, \varrho)$

- if $y-x \in X(x)$ then $\left\langle x^{\star}, y-x\right\rangle=0$ together with $\theta(y)=\theta(x)$ imply $\theta(y)+\sigma\|y-x\|^{2}=\theta(x)-\left\langle x^{\star}, y-x\right\rangle+\sigma\|y-x\| \geq \theta(x)$ and $x^{\star} \in \partial^{\mathrm{P}} \theta(x)$,

- otherwise, $y \neq x$ with $x^{\star} \in X(x)$; let $\underline{y}=\arg \min _{y \in B(x, \xi)} \theta(y)$ and $\underline{\sigma}=\max \left(0, \frac{\left\langle x^{\star}, y-x\right\rangle+\theta(x)-\theta(\underline{y})}{\|y-x\|}\right)$ which certainly exists. Therefore for all $\sigma>\underline{\sigma}$ we get $\theta(y)-\left\langle x^{\star}, y-x\right\rangle+\sigma\|y-x\| \geq \theta(x)$.

Theorem $2([8])$.

$$
\partial^{C} \theta(x)=X_{I^{\perp}}(x)
$$

Proof. From Theorem 1, $X_{I^{\perp}}(x) \subset \partial^{\mathrm{C}} \theta(x)$. 
- $\partial^{\mathrm{L}} \theta(x) \subset X_{I^{\perp}}(x)$.

Since $X_{I^{\perp}}(0)=\mathbb{R}^{n}$, we may assume $0 \neq x^{\star} \in \partial^{\mathrm{L}} \theta(x)$; by contradiction,

$$
\begin{aligned}
\liminf _{y \rightarrow x \neq y} \frac{\theta(y)-\theta(x)-\left\langle x^{\star}, y-x\right\rangle}{\|y-x\|}>0 \\
\left\langle x_{n}^{\star}, x_{n}-x\right\rangle=0
\end{aligned}
$$

since $x^{\star} \notin X_{I^{\perp}}(x)$ by contradictory assumption and $x_{n}^{\star} \in \partial^{\mathrm{F}} \theta\left(x_{n}\right)$ implies the latter by previous theorem. However, for $x \neq 0$ we have seen that for sufficiently small $\varrho$ and $x_{n} \in B(x, \varrho)$, we have $\theta\left(x_{n}\right)-\theta(x) \leq 0$ which violates first strict inequality. Overall $\partial^{\mathrm{L}} \theta(x)=X_{I^{\perp}}(x)$.

- $X_{I^{\perp}}(x)$ is convex; by definition, $x_{1}, x_{2} \in X_{I^{\perp}}(x)$ implies $\left(\alpha x_{1}+(1-\alpha) x_{2}\right) \in$ $X_{I^{\perp}}(x)$ and $\theta\left(\alpha x_{1}+(1-\alpha) x_{2}\right) \leq|I(x)|$ for $0 \leq \alpha \leq 1$.

- $\partial^{\mathrm{L} \infty} \theta(x)=\emptyset$.

Since projection of $X_{I^{\perp}}(x)$ on every $i \in I$ is $\operatorname{Pr}_{i}\left(X_{I^{\perp}}(x)\right)=\mathbb{R}$ is unbounded, let $\infty_{I} \in \mathbb{R}^{n}$ be the point whose components in $I$ are infinite. By abuse of notation, we may consider $\infty_{I} \in \partial^{\mathrm{L}} \theta(x)$ hence the emptyness of singular limiting subdifferential.

The result follows by convexity of $X_{I^{\perp}}(x)=\partial^{\mathrm{L}} \theta(x)$.

Let $e$ denote the all $1 \mathrm{~s}$ vector in $\mathbb{R}^{n}$ and $|\cdot|$ stands for the componentwise absolute value of a given vector.

\section{Lemma 1.}

$$
\left\langle e, \frac{|x|}{\|x\|}\right\rangle \leq \theta(x)=\|\operatorname{sgn}(x)\|^{2}
$$

Proof.

$$
x_{i}^{2} \leq x_{i}^{2}+\sum_{j \neq i} x_{j}^{2} \Rightarrow \frac{\left|x_{i}\right|}{\|x\|} \leq\left|\operatorname{sgn}\left(x_{i}\right)\right|
$$

In [10, 11] the authors proved that $f \circ \sigma$ is lower semicontinuous around the matrix $M \in \mathbb{R}^{m \times n}$ for an absolute symmetric function $f$, which is lower semicontinuous around the singular value vector $\sigma(M) \in \mathbb{R}^{\min (m, n)}$. Using this result, one can calculate a subdifferential for the rank function thanks to its decomposition $\theta \circ \sigma$ (see [8]). It could be specialized to symmetric matrices $M$ around eigenvalues under, say decreasing order for the uniqueness of orthogonal matrix $U$ such that $M=U \lambda(M) U^{-1}$ (see [6, 9]).

\section{More SUbdifferentials}

By discarding local conditions in viscosity subdifferential, several authors (see 12] and references therein) proposed a natural extension of both subdifferential and normal cone.

Definition 1. Let $\mathcal{L}$ be a set of real valued and continuous functions $l: \mathbb{R}^{n} \rightarrow$ $\mathbb{R} \cup \pm \infty$

$$
\begin{aligned}
\partial^{S} f(x) & =\left\{l \in \mathcal{L} \mid f(x+d) \geq f(x)+l(x+d)-l(x), \text { for all } d \in \mathbb{R}^{n}\right\}, \\
\mathrm{N}^{S}(x ; D) & =\{l \in \mathcal{L} \mid l(x+d)-l(x) \leq 0, \text { for all } d \in D-x\},
\end{aligned}
$$


where $d \in D-x$ stands for $(x+d) \in D$ to stress the condition on the direction at a given point $x$.

In [1, 3, 7, 13, 14, 16, 15, for non convex optimization problems global optimality conditions are derived in terms of $\partial^{\mathrm{S}} f(x), \mathrm{N}^{\mathrm{S}}(x ; D)$.

Recently, the authors [4] extended above subdifferential further by decoupling the domain space from the directional change rather than composing it along some direction and obtained necessary and sufficient conditions for global optimality too.

Definition 2 (Q-subdifferential). Let $\mathcal{Q}$ be a set of real valued functions $q: \mathbb{R}^{n} \times$ $\mathbb{R}^{p} \rightarrow \mathbb{R} \cup \pm \infty$ continuous in the first argument and let a map $\pi: \mathbb{R}^{p} \rightarrow \mathbb{R}^{n}$

$$
\begin{aligned}
\partial^{Q} f(x) & =\left\{q \in \mathcal{Q} \mid f(x+\pi(d)) \geq f(x)+q(x, d)-q(x, 0), \text { for all } d \in \mathbb{R}^{p}\right\} \\
\mathrm{N}^{Q}(x ; D) & =\{q \in \mathcal{Q} \mid q(x, d)-q(x, 0) \leq 0, \text { for all } \pi(d) \in D-x\}
\end{aligned}
$$

An element $q \in \partial^{\mathrm{Q}} f(x)$ is called a Q-subgradient of $f$ at $x$ and $\pi(d) \in D-x$ stands for $(x+\pi(d)) \in D$ in Q-normal cone definition. However, in the lack of well accepted terminology, the image of a Q-subgradient is improperly refered to as a Q-subdifferential (as much as in the S-case given by Rubinov) until a right naming is widely accepted.

Clearly, for $q_{1}, q_{2} \in \partial^{\mathrm{Q}} f(x)$ and $\alpha \in[0,1]$ the following inequalities hold

$$
\begin{aligned}
\alpha f(x+\pi(d)) & \geq \alpha\left(f(x)+q_{1}(x, d)-q_{1}(x, 0)\right) \\
(1-\alpha) f(x+\pi(d)) & \geq(1-\alpha)\left(f(x)+q_{2}(x, d)-q_{2}(x, 0)\right) \\
f(x+\pi(d)) & \geq f(x)+\left(\alpha q_{1}+(1-\alpha) q_{2}\right)(x, d)-\left(\alpha q_{1}+(1-\alpha) q_{2}\right)(x, 0)
\end{aligned}
$$

which proves the convexity of $\partial^{\mathrm{Q}} f(x)$.

Observe that for the special case $\pi=\mathbf{i d}$, the identity, we get

$$
\partial^{\mathrm{Q}} f(x)=\left\{q \in \mathcal{Q} \mid f(x+d) \geq f(x)+q(x, d)-q(x, 0), \text { for all } d \in \mathbb{R}^{n}\right\}
$$

and moreover $\partial^{\mathrm{S}} f(x) \subset \partial^{\mathrm{Q}} f(x)$ by choosing simply $l(y)=q(\cdot, y)$.

In order to relate both subdifferentials to Clarke subdifferential, let us recall the standard and extended directional derivatives relative to the direction $d \in \mathbb{R}^{n}$

$$
\begin{aligned}
f^{\prime}(x ; d) & =\lim _{t \rightarrow 0^{+}} \frac{f(x+t d)-f(x)}{t} \\
f^{\mathrm{C}}(x ; d) & =\limsup _{y \rightarrow x, t \rightarrow 0^{+}} \frac{f(y+t d)-f(y)}{t} \\
f^{\mathrm{S}}(x ; d) & =\sup \left\{l(x+d)-l(x) \mid l \in \partial^{\mathrm{S}} f(x)\right\} \\
f^{\mathrm{Q}}(x ; d) & =\sup \left\{q(x, d)-q(x, 0) \mid q \in \partial^{\mathrm{Q}} f(x)\right\}
\end{aligned}
$$

so that

$$
\begin{aligned}
\xi \partial^{\mathrm{C}} f(x) \Longleftrightarrow\langle\xi, x+d\rangle-\langle\xi, x\rangle \leq f^{\mathrm{C}}(x ; d), \quad \forall d \in \mathbb{R}^{n} \\
l \in \partial^{\mathrm{S}} f(x) \Longleftrightarrow l(x+d)-l(x) \leq f^{\mathrm{S}}(x ; d), \quad \forall d \in \mathbb{R}^{n} \\
q \in \partial^{\mathrm{Q}} f(x) \Longleftrightarrow q(x, d)-q(x, 0) \leq f^{\mathrm{Q}}(x ; \pi(d)), \quad \forall d \in \mathbb{R}^{p}
\end{aligned}
$$

Proposition 1. Let suppose $f^{S}(x ; d)$ be positive subhomogeneous in $d$, namely $f^{S}(x ; t d) \leq t f^{S}(x ; d)$ for all $t>0$, then

$$
\begin{gathered}
l \in \partial^{S} f(x) \Rightarrow f^{C}(x ; d) \leq l^{\prime}(x ; d) \\
\partial^{C} f(x) \subset \partial^{S} f(x)
\end{gathered}
$$


Proof. Let $g(x)=l(x+t d)-l(x)$, we apply the Ekeland principle for $g$, not identically 0 and bounded by below, such that $g(x) \leq \inf g(x)+\epsilon$ for $\epsilon>0$, to get

$$
\begin{aligned}
\exists v, \lim _{t \rightarrow 0^{+}} \frac{g(x)}{t} & \geq \lim _{v \rightarrow x, t \rightarrow 0^{+}} \frac{g(v)}{t} \\
& \geq \limsup _{v \rightarrow x, t \rightarrow 0^{+}} \frac{f(v+t d)-f(v)}{t}=f^{\mathrm{C}}(x ; d)
\end{aligned}
$$

where we select the direction $d$ such that $x=v+t d$ so that $f(v+t d)-f(v) \leq$ $l(v+t d)-l(v)$ since $l \in \partial^{\mathrm{S}} f(x)$. Then, by assumption $f^{\mathrm{S}}(x ; d) \geq \lim _{t \rightarrow 0^{+}} \frac{g(x)}{t}$ for all $l \in \partial^{\mathrm{S}} f(x)$, hence we conclude $f^{\mathrm{S}}(x ; d) \geq f^{\mathrm{C}}(x ; d)$ and the expected inclusion is proven.

\section{Remark 2.}

(1) If $l^{\prime}(x ; d)$ is weakened to $l^{C}(x ; d)$ instead, then the result holds without the Ekeland principle and we retrieve the sublinear property that may fail for $l^{\prime}(x ; d)$ provided $f^{S}(x ; d) \geq l^{C}(x ; d)$.

(2) For locally Lipschitz function $f$, in the Frechet setting we have more directly

$$
\partial^{C} f(x)=\operatorname{conv}\left\{\lim \nabla f\left(x_{k}\right) \mid x_{k} \rightarrow x, x_{k} \notin \Omega_{f}, x_{k} \notin S\right\}
$$

Let $l(x)=\sigma\left\|x-x_{k}\right\|^{2}+\min \left\{\left\langle\nabla f\left(x_{k}\right), x-x_{k}\right\rangle\right\}$ where the minimum is over all linear functions $\left\langle\nabla f\left(x_{k}\right), x-x_{k}\right\rangle$ with $\nabla f\left(x_{k}\right) \in \partial^{C} f(x)$ then $f(x) \leq f\left(x_{k}\right)+l(x)-l\left(x_{k}\right)$ for some $\sigma>0$ and $\partial^{C} f(x) \subset \partial^{S} f(x)$.

Proposition 2. Let us assume that $q(x, \cdot) \in \mathcal{Q}$ be lower semi-continuous and $f^{Q}(x ; d)$ be positive subhomogeneous, both in the second argument, then

$$
\begin{aligned}
q \in \partial^{Q} f(x) & \Rightarrow f^{C}(x ; \pi(d)) \leq q_{x, 0}^{\prime}(x ; d) \\
\partial^{C} f(x) & \subset \partial^{Q} f(x)
\end{aligned}
$$

where $q_{x, 0}^{\prime}(x ; d)=\lim _{t \rightarrow 0^{+}} \frac{q(x, t d)-q(x, 0)}{t}$ is a directional derivative at 0 , with respect to the second argument.

Proof. Let $h(x)=q(x, t d)-q(x, 0)$; we apply the Ekeland principle for $h$, not identically 0 and bounded by below, such that $h(x) \leq \inf h(x)+\epsilon$ for $\epsilon>0$, to get

$$
\begin{aligned}
\exists y, \lim _{t \rightarrow 0^{+}} \frac{h(x)}{t} & \geq \lim _{y \rightarrow x, t \rightarrow 0^{+}} \frac{h(y)}{t} \\
& \geq \limsup _{y \rightarrow x, t \rightarrow 0^{+}} \frac{f(y+t \pi(d))-f(y)}{t}=f^{\mathrm{C}}(x ; \pi(d))
\end{aligned}
$$

Using the assumption $f^{\mathrm{Q}}(x ; t d) \leq t f^{\mathrm{Q}}(x ; d)$, we conclude $f^{\mathrm{Q}}(x ; d) \geq f^{\mathrm{C}}(x ; \pi(d))$ and the expected inclusion is proven under mild assumptions on $\pi$.

Remark 3. Like in the $\partial^{S} f(x)$ case, the Ekeland principle could be skipped, provided the subhomogeneity is replaced by a decoupled Clarke's derivative in the first argument at $x$ and a directional derivative in the second argument at 0 $f^{Q}(x ; d) \geq \lim _{y \rightarrow x, t \rightarrow 0^{+}} \frac{q(y, t d)-q(y, 0)}{t} \simeq q_{x, 0}^{C}{ }^{\prime}(x ; d)$.

There is no obvious composition rule for both subdifferentials unless the functions in $\partial f(x)$ have a representation in $\mathbb{R}^{n}$. 


\section{The SIGN CHANGE COUNTING FUnCtion}

The number of sign transitions along the components may be seen as a first order derivative of the sign counting function; its active index set is defined accordingly

$$
\begin{aligned}
J(x) & =\left\{i \mid \operatorname{sgn}\left(x_{i}\right)=\operatorname{sgn}\left(x_{i+1}\right)\right\} \\
J^{\|}(x) & =\{i, i+1 \mid i \in J(x))\}, \quad J^{\perp}(x)=\left\{i \mid i \notin J^{\|}(x)\right\} \\
X_{J \|}(x) & =\left\{y \in \mathbb{R}^{n} \mid \operatorname{sgn}\left(y_{i}\right)=\operatorname{sgn}\left(x_{i}\right) \text { for all } i \in J^{\|}(x)\right\} \\
\tau(x) & =\left|J^{\perp}(x)\right|
\end{aligned}
$$

where $i+1$ is possibly taken modulo $n$ for circular counting (as required in some real life applications). For instance, at $x=(-24,-30,19,14,0)^{\top} \in \mathbb{R}^{5}$ the sign change counting function output is $\tau(-1,-1,1,1,0)=3$.

Lemma 2. Let assume $x_{n+1}=x_{1}, x_{n}$ according with circular case or not, and define $l_{i}(x ; k)=\left(\operatorname{sgn}\left(x_{i}\right)+\operatorname{sgn}\left(x_{i+1}\right)+k \operatorname{sgn}\left(x_{i} x_{i+1}\right)\right)\left(\operatorname{sgn}\left(x_{i} x_{i+1}\right)-1\right)$ for the parameter $k$ then $l_{i}(x ; k)=0$ iff $i \in J(x)$ provided $k \neq 0$. Moreover, $\left|l_{i}(x ; 1)\right|$ measures the gap between the 2 consecutive components.

Proof. Observe that $l_{i}(x ; k)=\left(k+2 \operatorname{sgn}\left(x_{i}\right)\right)(1-1)=0$ whenever $x_{i+1}=x_{i}$ to deal with non circular case and no sign change, then by enumeration of remaining cases,

$$
l_{i}(x ; k)=\left\{\begin{array}{l}
(-1+0+0 k)(0-1)=1 \\
(-1+1-k)(-1-1)=-2 k \\
(0-1-0 k)(0-1)=1 \\
(0+1+0 k)(0-1)=-1 \\
(1-1-k)(-1-1)=2 k \\
(1+0+0 k)(0-1)=-1
\end{array}\right.
$$

As a direct consequence, since $l_{i}(0 ; k)=0$ for all $i$

Corollary 1. Let $l: \mathbb{R}^{n} \rightarrow \mathbb{R}^{n}$ be the map defined by

$$
l(x ; k)=\left[\begin{array}{lll}
l_{1}(x ; k) & \ldots & l_{n}(x ; k)
\end{array}\right]^{\top}
$$

then $l(0 ; k)=0$ and

$$
\theta(l(x ; 0<|k|<1 / 2)) \leq \tau(x)=\theta(l(x ; \pm 1 / 2))=\|l(x ; \pm 1 / 2)\|^{2} \leq \theta(l(x ; 1 / 2<|k|))
$$

since the absolute value emphasizes a sign change for $k= \pm 1 / 2$, while it is respectively minorized and majorized otherwise. Notice that the parameter is only involved in \pm 1 sign transitions and $k= \pm 1$ amplifies the sign change count for such transitions by a factor 2 , therfeore $|k|=1$ play the role of an upper bound somehow. A smoothed minorant version directly follows the smoothed version of the sign counting function

$$
\left\{\begin{array}{l}
\tau(x) \geq \tau_{\epsilon}(x)=\theta_{\epsilon}\left(l\left(x ; \pm \frac{1}{2}\right)\right) \\
\lim _{\epsilon \rightarrow 0} \tau_{\epsilon}(x)=\tau(x) .
\end{array}\right.
$$

For $n=2$ the function $\tilde{l}_{1}: \mathbb{R}^{2} \rightarrow \mathbb{R}$ defined by

$$
\tilde{l}_{1}(x ; \pm 1 / 2)=\left(x_{1}+x_{2} \pm x_{1} x_{2} / 2\right)\left(x_{1} x_{2}-1\right)
$$

one can calculate the hessian

$$
\nabla^{2} \tilde{l}_{1}(x ; \pm 1 / 2)=\left[\begin{array}{cc}
x_{2}\left(2 \pm x_{2}\right) & 2\left(\mp 1 / 4+x_{1}+x_{2} \pm x_{1} x_{2}\right) \\
2\left(\mp 1 / 4+x_{1}+x_{2} \pm x_{1} x_{2}\right) & x_{1}\left(2 \pm x_{1}\right)
\end{array}\right]
$$


with eigenvalues at $\operatorname{sgn}(x)$ points, with opposite signs (which means $\tilde{l}_{1}$ neither convex nor concave)

$$
\begin{array}{r}
2 \Lambda\left[\begin{array}{ll}
-1 & -1
\end{array}\right]^{t}=\mp 2 \pm 5 \\
2 \Lambda\left[\begin{array}{ll}
0 & 0
\end{array}\right]^{t}= \pm 1 \\
2 \Lambda\left[\begin{array}{ll}
+1 & +1
\end{array}\right]^{t}= \pm 6 \mp 11 \\
2 \Lambda\left[\begin{array}{ll}
-1 & 0
\end{array}\right]^{t}=2 \Lambda\left[\begin{array}{ll}
0 & -1
\end{array}\right]^{t}=\mp 1 \pm \sqrt{26} \\
2 \Lambda\left[\begin{array}{ll}
-1 & +1
\end{array}\right]^{t}=2 \Lambda\left[\begin{array}{ll}
+1 & -1
\end{array}\right]^{t}= \pm 2 \pm \sqrt{41} \\
2 \Lambda\left[\begin{array}{ll}
0 & +1
\end{array}\right]^{t}=2 \Lambda\left[\begin{array}{ll}
+1 & 0
\end{array}\right]^{t}= \pm 3 \pm 3 \sqrt{2}
\end{array}
$$

so that for all $i, \tilde{l}_{i}(x ; \pm 1 / 2)$ is twice differentiable at $\operatorname{sgn}(x)$.

Under a slight abuse of notation, where $\|l(x ; \pm 1 / 2)\|^{2}$ stands for $l(x)$, and under the special transform $\pi=$ id we prove our main result of the section.

\section{Theorem 3.}

$$
\begin{aligned}
\|l(x ; \pm 1 / 2)\|^{2} & \in \partial^{S} \tau(x) \\
q\left(x, y ; k_{x}, k_{y}\right)-q\left(x, 0 ; k_{x}, k_{y}\right) & =\left\|l\left(x+y ; 0<\left|k_{y}\right| \leq 1 / 2\right)\right\|^{2}-\left\|l\left(x ; 1 / 2 \leq\left|k_{x}\right|\right)\right\|^{2} \\
q\left(x, y ; k_{x}, k_{y}\right) & \in \partial^{Q} \tau(x)
\end{aligned}
$$

Proof. $\tau(x)=\tau(y)-\|l(y, \pm 1 / 2)\|^{2}+\|l(x ; \pm 1 / 2)\|^{2}$ leads to the coupled case $\partial^{\mathrm{S}} \tau(x)$; however, it does not help much as a direction for minimizing the number of sign changes, as a neutral subgradient. The decoupled case follows by applying Corollary 1, $\tau(y)-\tau(x) \geq\left\|l\left(y ; 0<\left|k_{y}\right| \leq 1 / 2\right)\right\|^{2}-\left\|l\left(x ; 1 / 2 \leq\left|k_{x}\right|\right)\right\|^{2}$.

Notice that

$$
\begin{aligned}
& \hat{q}\left(x, 0 ; 0<\left|k_{y}\right| \leq 1 / 2 \leq\left|k_{x}\right|\right)=\left\|l\left(x ; 0<\left|k_{y}\right| \leq 1 / 2\right)\right\|^{2}-\left\|l\left(x ; 1 / 2 \leq\left|k_{x}\right|\right)\right\|^{2} \\
& \quad=\left(k_{y}-k_{x}\right) \sum\left(x_{i} x_{i+1}-1\right)^{2}\left(\left(k_{y}+k_{x}\right) x_{i}^{2} x_{i+1}^{2}+2 x_{i} x_{i+1}\left(x_{i}+x_{i+1}\right)\right)<0
\end{aligned}
$$

where for sake of brevity, we write $x_{i}$ instead of $\operatorname{sgn}\left(x_{i}\right)$; clearly, each summand accounts for 0 if either one component is 0 , or both components have same sign, and for $4\left(k_{y}^{2}-k_{x}^{2}\right)<0$ otherwise. Therefore

$$
\begin{aligned}
\tau(y)-\tau(x) & \geq \hat{q}\left(x, y-x ; 0<\left|k_{y}\right| \leq 1 / 2 \leq\left|k_{x}\right|\right) \\
& \geq \hat{q}\left(x, y-x ; 0<\left|k_{y}\right| \leq 1 / 2 \leq\left|k_{x}\right|\right)+\hat{q}\left(x, 0 ; 0<\left|k_{y}\right| \leq 1 / 2 \leq\left|k_{x}\right|\right)
\end{aligned}
$$

showing that $q$ is implicitly known. However, it improves over $\partial^{\mathrm{S}} \tau(x)$ with the parameters $k_{y}, k_{x}$ varying.

Remark 4. In a domain $D$ in the vicinity of $x$, parameters $k_{x}$ and $k_{y}$ may vary such that $q\left(x, d ; k_{x}, k_{y}\right) \in \mathrm{N}^{Q}(x ; D)$. It would be interesting to check whether a similar situation arises for $\mathrm{N}^{S}(x ; D)$ for a single parameter $k$ varying.

Remark 5. Let I (resp. Z) be the identity (resp. circular shift) matrix conformably to the vector size $x$ and $\odot$ the componentwise (Hadamard) product; the $i$-th power composition of $\odot$ is denoted by a self explanatory exponent ()$^{\odot} i$. In this algebraic writing, the relationship $\|x \odot y\|^{2}=\left\langle x^{\odot 2}, y^{\odot 2}\right\rangle$ gives

$$
\|l(x ; k)\|^{2}=\left\langle((I+Z) x+k(x \odot Z x))^{\odot 2},((x \odot Z x)-e)^{\odot 2}\right\rangle
$$


with the all $1 s$ vector $e$.

Lemma 3. For all $x \notin \arg \operatorname{loc} \min t$ and $x \neq 0$

$$
\left\{\begin{array}{l}
\partial^{F} \tau(x) \subset X^{\perp}(x), x \in \arg \operatorname{loc} \max t \\
\partial^{F} \tau(x)=0, \text { otherwise }
\end{array}\right.
$$

Proof. Frechet differential is computed, in the same way as for the sign function for simple cases

- no 0 component in the vicinity of $x$. For all $x+\tau y \in B(x, \varrho), \tau(x+\tau y)=$ $\tau(x)$, hence $\left\langle x^{\star}, y\right\rangle \leq 0$ for all $x^{\star} \in \partial^{\mathrm{F}} \tau(x)$. Then, suppose $\left\langle x^{\star}, y\right\rangle<0$ and consider $\bar{y}=-y$ to arrive at the contradiction $\left\langle x^{\star}, \bar{y}\right\rangle>0$ for $x^{\star} \in \partial^{\mathrm{F}} \tau(x)$. Finally, suppose $x_{j}^{\star} \neq 0$ for some $j$ and $\sum x_{i}^{\star} y_{i}=0$, since there is no sign change in $B(x, \varrho)$ taking $\bar{y}=y$ except for taking the opposite of $y_{j} \neq 0$, we get $\sum x_{i}^{\star} \bar{y}_{i}=-2 x_{j}^{\star} y_{j} \neq 0$. It yields $\partial^{\mathrm{F}} \tau(x)=0$ and we consider $x$ to be stationary.

- $X^{\perp}(0)=\mathbb{R}^{n} \supset \partial^{\mathrm{F}} \tau(0)$.

- $x \neq 0$ is a local maximum. For all $x+\tau y \in B(x, \varrho), \tau(x+\tau y) \leq \tau(x)$, and we get $\left\langle x^{\star}, y\right\rangle=0$, provided $x^{\star} \neq 0$ to derive both previous contradictions. Therefore $\partial^{\mathrm{F}} \tau(x) \subset X^{\perp}(x)$.

If $0 \neq x \in \arg \operatorname{loc} \min t$ then $\partial^{\mathrm{F}} \tau(x)$ raises combinatorial enumeration depending on the number of \pm 1 signs; this issue does not arise for $\partial^{\mathrm{Q}} \tau(x)$.

As an example, consider the Q-subdifferential $\tau(x)$ at a given transition point $x$ and direction $d$

$$
q^{\prime}(x ; d)=-310+692 k-888 k^{2}\left\{\begin{array}{l}
x=\left[\begin{array}{lllllll}
-1 & 1 & 1 & 0 & -1 & 0 & 0
\end{array}\right]^{t} \\
d=\left[\begin{array}{lllllll}
0 & 74 & 75 & 0 & -40 & -50 & 0
\end{array}\right]^{t}
\end{array}\right.
$$

for the varying parameter $0<k \leq 1 / 2$, since the parameter $1 / 2 \leq k_{x}$ does not play any role in this instance Figure 1 . The quadratic term justifies the naming of subdifferential.

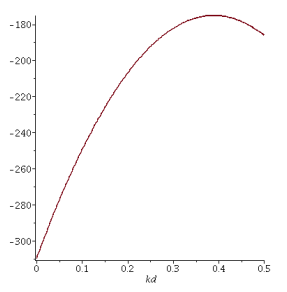

Figure 1. example of $\partial^{\mathrm{Q}} \tau(x)$ for varying parameter $0<k \leq 1 / 2$

5.1. Example of the continuous multipliers. Let us remind, to be self contained, the sufficient condition for global minimizer established by the authors under the identity map $\pi=\mathbf{i d}: \mathbb{R}^{n} \rightarrow \mathbb{R}^{n}$ 
Proposition 3 (4]). Let $Q$ be a set of real valued functions such that $-q \in Q$ for $q \in Q$; if there are continuous multipliers $\lambda_{i}(x) \geq 0$ for all $x \in \mathcal{F}$ and a feasible $z \in \mathcal{F}$ fulfilling

$$
\begin{aligned}
& 0 \in \partial^{Q} f(z)+\partial^{Q}\left(\sum_{i \in M} \lambda_{i} g_{i}\right)(z), \\
& \left(\sum_{i \in M} \lambda_{i} g_{i}\right)(z)=0
\end{aligned}
$$

then $z$ is a global minimizer for $\min f(x)$ under $g_{i}(x) \leq 0$.

We considered in [4] the following example from [3].

$$
\begin{aligned}
& \min x^{2} \cos (2 x) \\
& \text { s.t. }\left\{\begin{array}{l}
-x-2 \pi \leq 0 \\
x-2 \pi \leq 0
\end{array}\right.
\end{aligned}
$$

Choose multipliers $\lambda_{1}(x)=\left|x-c_{1}\right| \exp \left|x-c_{1}\right|, \lambda_{2}(x)=0$ and

$$
q_{f}(x, y ; \sigma)=(x+y)^{2} \cos (2(x+y))-y^{2} \cos (2 y)-\sigma|y| .
$$

We get for $c_{1}=-4.8, \sigma=1$ the system of dependent constraints

$$
\left\{\begin{array}{l}
-|x+4.8| \exp (|x+4.8|)(x+2 \pi)+x^{2} \cos (2 x)+22.687 \geq 0 \\
-|x+4.8| \exp (|x+4.8|)(x+2 \pi)-x^{2} \cos (2 x)-22.687+\sigma|x+4.8| \geq 0 \\
2 x^{2} \cos (2 x)+45.374-\sigma|x+4.8| \geq 0
\end{array}\right.
$$

which is fulfilled on the interval $[-2 \pi, 0]$ (see Figure 2), hence the global minimizer at $z=-4.8$.
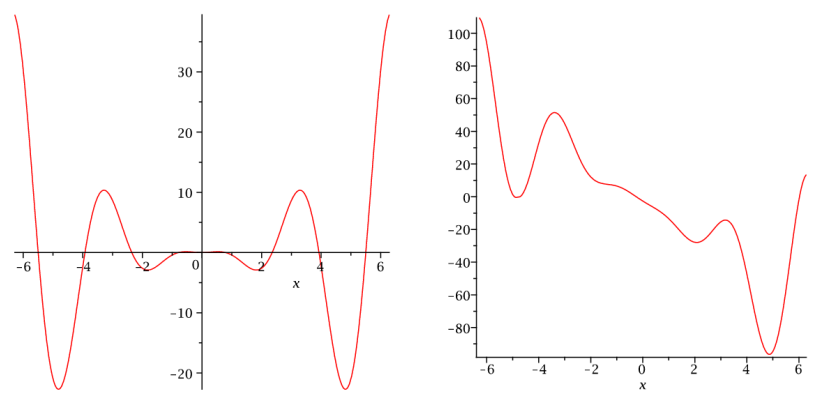

Figure 2. global minimizer at $z=-4.8$ : on the left $f$; on the right $\lambda_{1}$ multiplier with $\sigma=1$

5.2. Example showing some optimality condition checking difficulties. Splitting equality constraints $g_{i}(x)=x_{i}\left(1-x_{i}^{2}\right)=0$ which capture sign values $\{0, \pm 1\}$, into $g_{i}(x) \leq 0$ and $-g_{i}(x) \leq 0$, then sufficient optimality condition Proposition 3 for $z$ to be a global minimizer of $t$ simplifies by merging both non negative continuous multipliers into a single one without the non negative condition

$$
0 \in \partial^{\mathrm{Q}} t(z)+\partial^{\mathrm{Q}}\left(\sum_{i \in M} \lambda_{i} g_{i}\right)(z)
$$




$$
\begin{aligned}
\|l(z ; 1 / 2)\|^{2}-\|l(d ; k)\|^{2} & \in \partial^{\mathrm{Q}} t(z) \\
\left\langle\nabla\left(\lambda_{i} g_{i}\right)(z), d\right\rangle & =\left\langle\lambda \odot\left(e-3 z^{\odot 2}\right), d\right\rangle \in \partial^{\mathrm{Q}}\left(\sum_{i \in M} \lambda_{i} g_{i}\right)(z)
\end{aligned}
$$

since for all $i, j$ we have $z_{j}\left(1-z_{j}\right)^{2} \partial \lambda_{i} / \partial x_{i}=0$ at $z$.

5.2.1. trivial examples upto dimension 3. For the, obviously optimal, solution, $z=$ $[-1,1]$, it leads, under the circular case, to the equation:

$$
\begin{aligned}
\lambda_{1} d_{1}\left(1-3 z_{1}^{2}\right)+\lambda_{2} d_{2}\left(1-3 z_{2}^{2}\right)+2- & \left(\left(d_{1}+d_{2}+k_{12} d_{1} d_{2}\right)^{2}\right. \\
& \left.+\left(d_{1}+d_{2}+k_{21} d_{1} d_{2}\right)^{2}\right)\left(d_{1} d_{2}-1\right)^{2}=0
\end{aligned}
$$

w.l.o.g. we may select $k_{21}=k_{12}$ and polar coordinates $d_{1}=\cos \phi_{1}, d_{2}=\sin \phi_{1}$. There exists $\lambda_{1}=0$ and $k_{12}=0$ such that

$$
4 \lambda_{2} \sin \phi_{1}=4-\left(\cos \phi_{1}+\sin \phi_{1}\right)^{2}\left(\sin \left(2 \phi_{1}\right)-2\right)^{2}
$$

Symmetry associated with circular case allows us to reduce $d_{2}$ to non negative values, equivalently $\phi_{1} \in[0, \pi]$, then the sufficient condition is fulfilled as could be checked on Figure 3.

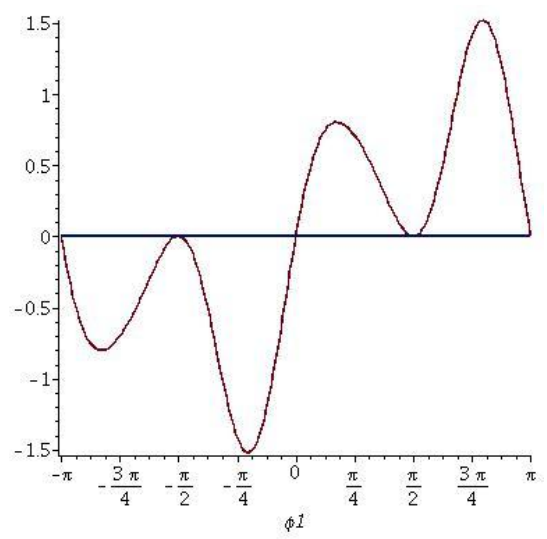

FiguRE 3. sufficient condition at $z=[-1,1] \lambda_{2}\left(\phi_{1}\right)$ under $\lambda_{1}=0$

For $z=[1,-1,1]$, with 2 sign changes, it leads to the equation:

$$
\begin{gathered}
0=\lambda_{1} d_{1}\left(1-3 z_{1}^{2}\right)+\lambda_{2} d_{2}\left(1-3 z_{2}^{2}\right)+\lambda_{3} d_{3}\left(1-3 z_{3}^{2}\right)+2-\left(d_{1}+d_{2}+k_{12} d_{1} d_{2}\right)^{2}\left(d_{1} d_{2}-1\right)^{2} \\
-\left(d_{2}+d_{3}+k_{23} d_{2} d_{3}\right)^{2}\left(d_{2} d_{3}-1\right)^{2}-\left(d_{3}+d_{1}+k_{31} d_{3} d_{1}\right)^{2}\left(d_{3} d_{1}-1\right)^{2}
\end{gathered}
$$

W.l.o.g. let us choose a spherical parametrization $d_{1}=\cos \phi_{1}, d_{2}=\cos \phi_{2} \sin \phi_{1}$, and $d_{3}=\sin \phi_{2} \sin \phi_{1}$; for symmetry reason once more, we may assume $d_{3} \geq 0$ so that $\phi_{1}, \phi_{2}$ shrink to the open interval $(0, \pi)$. Select $\lambda_{1}(x)=\lambda_{2}(x)=0$ and $k_{12}=k_{23}=k_{31}=0$, then

$$
\begin{aligned}
8 \lambda_{3} \sin \phi_{1} \sin \phi_{2}=8 & -\left(\cos \phi_{1}+\cos \phi_{2} \sin \phi_{1}\right)^{2}\left(\cos \phi_{2} \sin \left(2 \phi_{1}\right)-2\right)^{2} \\
& -\sin ^{2} \phi_{1}\left(\cos \phi_{2}+\sin \phi_{2}\right)^{2}\left(\sin ^{2} \phi_{1} \sin \left(2 \phi_{2}\right)-2\right)^{2} \\
& -\left(\cos \phi_{1}+\sin \phi_{1} \sin \phi_{2}\right)^{2}\left(\sin \phi_{2} \sin \left(2 \phi_{1}\right)-2\right)^{2}
\end{aligned}
$$



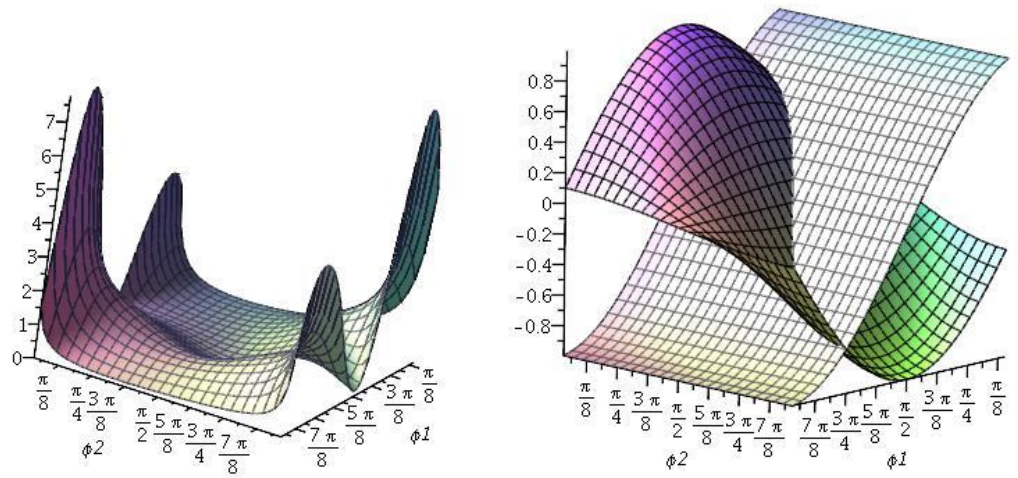

Figure 4. sufficient condition at $z=[1,-1,1] \lambda_{3}\left(\phi_{1}, \phi 2\right)$ under $\lambda_{1}=\lambda_{2}=0$ (left) and directions $d_{1}, d_{2}$ (right)

5.2.2. first non trivial example. A careful look at Equation 9 reveals that, on the contrary to 1-dimensional example subsection 5.1 that no explicit continuous multipliers are able to fulfill the sufficient condition since, on the one hand $\|l(z ; 1 / 2)\|^{2}-\|l(d ; k)\|^{2}=0$ whenever $|k|=\frac{1}{2}$ so that at least one component in left hand side has to be not 0 or else every point will be optimal, a contradiction, and, on the other hand, the left hand side leads to a system of $3^{4}-1$ equations in 4 parameters (whatever there relationship with the multiplier functions). Until the sufficient condition is extended to a different case than identity mapping, the hope remains to find a stronger condition that would be fulfilled at optimal points without constructing the multipliers (implicitly at most).

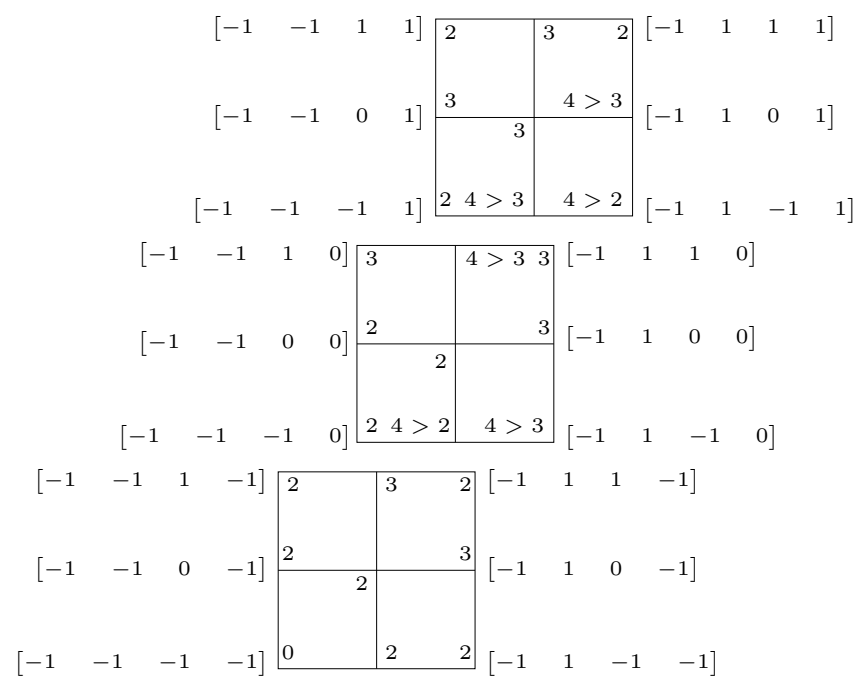

FiguRE 5. sign changes at $z_{1}=-1$ 
GENERALIZED SUBDIFFERENTIALS OF THE SIGN CHANGE COUNTING FUNCTION 13

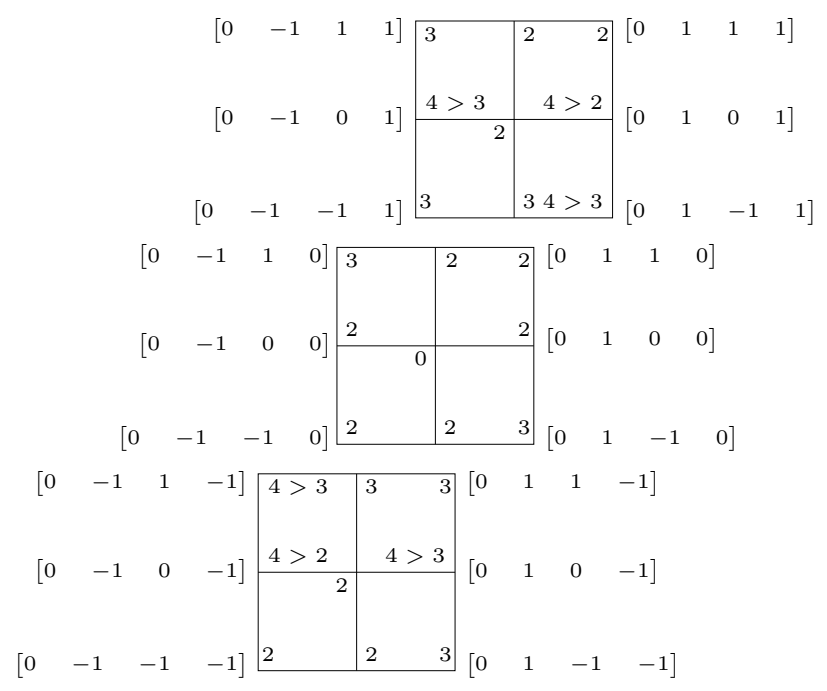

FiguRE 6. sign changes at $z_{1}=0$

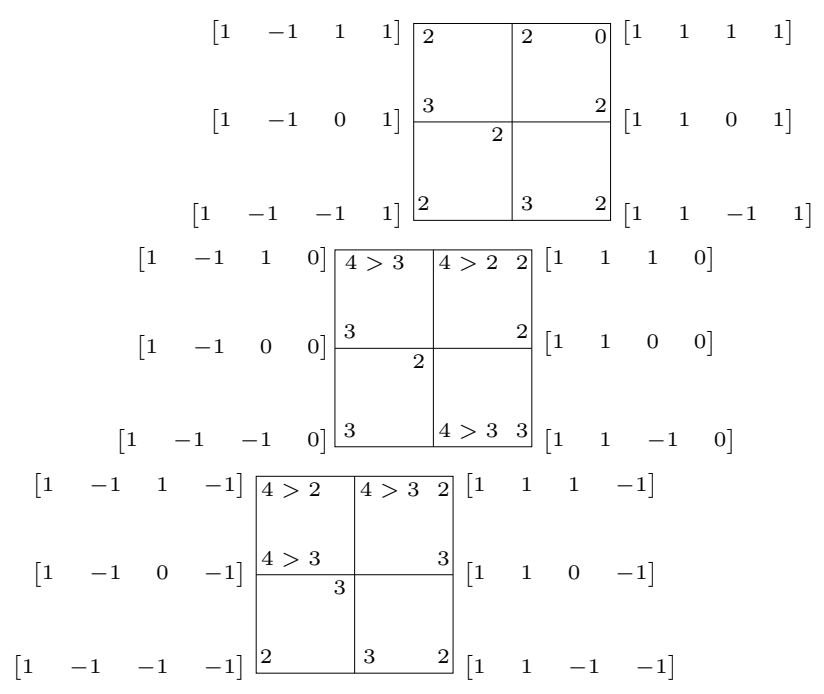

Figure 7. sign changes at $z_{1}=1$

By enumeration, we can see from Figure 5, Figure 6, and Figure 7 that not optimal points, $t(z)>3$, are symmetric with respect to center $[0,0,0,0]$.

It suggests to consider spherical coordinates and gradient of a scalar function in spherical coordinates:

$$
x=\left[\begin{array}{c}
\rho \cos \phi_{1} \\
\rho \cos \phi_{2} \sin \phi_{1} \\
\rho \cos \phi_{3} \sin \phi_{1} \sin \phi_{2} \\
\rho \sin \phi_{3} \sin \phi_{1} \sin \phi_{2}
\end{array}\right], \quad \nabla f=\left[\begin{array}{c}
f^{(\rho)} \\
f^{\left(\phi_{1}\right)} / \rho \\
f^{\left(\phi_{2}\right)} / \rho \sin \phi_{1} \\
f^{\left(\phi_{3}\right)} / \rho \sin \phi_{1} \sin \phi_{2}
\end{array}\right]
$$


where $f^{(\xi)}$ stands for $\frac{\partial f}{\partial \xi}$ for sake of shortness. Let us suppose for checking the stronger condition at $z$ that

$$
\partial^{\mathrm{Q}}\left(\sum_{i \in M} \lambda_{i} g_{i}\right)(z)=\left[\begin{array}{llll}
\mu_{1} & \mu_{2} & \mu_{3} & \mu_{4}
\end{array}\right]^{t}
$$

then we have to check whether there exists a solution to the polynomial equations

$$
\begin{aligned}
t(z)=\mu_{1} & (z) \rho c_{1}+\mu_{2}(z) \rho c_{2} s_{1}+\mu_{3}(z) \rho c_{3} s_{1} s_{2}+\mu_{4}(z) \rho s_{1} s_{2} s_{3} \\
& +\left(\rho c_{1}+\rho s_{1} s_{2} s_{3}\right)^{2}\left(\rho^{2} c_{1} s_{1} s_{2} s_{3}-1\right)^{2} \\
& +\left(\rho c_{1}+\rho c_{2} s_{1}\right)^{2}\left(\rho^{2} c_{1} c_{2} s_{1}-1\right)^{2} \\
& +\left(\rho c_{2} s_{1}+\rho c_{3} s_{1} s_{2}\right)^{2}\left(\rho^{2} c_{2} s_{1}^{2} c_{3} s_{2}-1\right)^{2} \\
& +\left(\rho c_{3} s_{1} s_{2}+\rho s_{1} s_{2} s_{3}\right)^{2}\left(\rho^{2} c_{3} s_{1}^{2} s_{2}^{2} s_{3}-1\right)^{2} \\
1=c_{i}^{2} & +s_{i}^{2}, \quad i=1 \cdots 3
\end{aligned}
$$

in the variables $\rho, c_{i}, s_{i}$ related to the modulus and cosine and sine of the spherical coordinates.

Notice that only feasible directions are required so that further conditions would be added to restrict spherical coordinates depending on $z$ to fit the 4-dimensional hypercube; in particular $\rho^{2} \in\{1,2,3,4,5,6,7,8,9,10,12,13,16\}$. Clearly, existence of a solution to either system of 4 non linear equations in 6 variables implies the true satisfaction of the original condition. To our knowledge, this example overtakes the recent advances in polynomial systems 2 both in dimension and degree and remains an opened challenging issue for the identity mapping; furthermore, should a positive answer given, no implicit definition of the multipliers will be found so that the promise of improving a not optimal point thanks to the subdifferential flies away.

\section{Concluding Remarks}

In this article, we introduce the sign change counting function as a natural extension of the sign counting function whose role is prominent in studying various set of matrices. Its Q-subdifferential is given and an attempt is made to prove a sufficient condition for global optimality at a point. The failure of this condition, which was specially tailored in the case of the identity map $\mathbb{R}^{n} \rightarrow \mathbb{R}^{n}$, raises the question of new sufficient conditions for lifted maps $\pi: \mathbb{R}^{p} \rightarrow \mathbb{R}^{n}$ with $p>n$ that afford checking for the inverse of some linear system in $p$ parameters related to lagrangian multipliers (after projection back into original space $\mathbb{R}^{n}$ ). We believe that the sign change counting function is interesting in itself and has furthermore, a fundamental impact in fully splitted spectrum of matrices as devised by our initial motivation. Last, it is worth mentioning, as a quite long term goal, the design of an optimal sort algorithm associated with a given vector; the almost ready made extension of this function (just think of $J(x)=\left\{i \mid x_{i}=x_{i+1}\right\}$ ), would lead to an algorithm that follows the Q-subdifferential to apply necessary and sufficient exchanges, on the contrary to general purpose sort algorithms.

\section{REFERENCES}

[1] D. Carrasco-Olivera and F. Flores-Bazán. On the representation of approximate subdifferentials for a class of generalized convex functions. Set-Valued Anal., 13(2):151-166, 2005. 
[2] M. Elkadi and B. Mourrain. Some Applications of Bezoutians in Effective Algebraic Geometry. Technical Report RR-3572, INRIA, Dec. 1998.

[3] F. Flores-Bazán. On minima of the difference of functions. J. Optim. Theory Appl., 93(3):525$531,1997$.

[4] D. Fortin and I. Tsevendorj. Q-subdifferential and Q-conjugate for global optimality. Comp. Math. Phys., (1), 2014. to appear.

[5] J.-B. Hiriart-Urruty and H. Y. Le. Convexifying the set of matrices of bounded rank: applications to the quasiconvexification and convexification of the rank function. Optim. Lett., 6(5):841-849, 2012.

[6] J.-B. Hiriart-Urruty and A. S. Lewis. The Clarke and Michel-Penot subdifferentials of the eigenvalues of a symmetric matrix. Comput. Optim. Appl., 13(1-3):13-23, 1999. Computational optimization - a tribute to Olvi Mangasarian, Part II.

[7] V. Jeyakumar, A. M. Rubinov, and Z. Y. Wu. Non-convex quadratic minimization problems with quadratic constraints: global optimality conditions. Math. Program., 110(3, Ser. A):521$541,2007$.

[8] H. Y. Le. Generalized subdifferentials of the rank function. Optim. Lett., 7(4):731-743, 2013.

[9] A. S. Lewis. Nonsmooth analysis of eigenvalues. Math. Program., 84(1, Ser. A):1-24, 1999.

[10] A. S. Lewis and H. S. Sendov. Nonsmooth analysis of singular values. I. Theory. Set-Valued Anal., 13(3):213-241, 2005.

[11] A. S. Lewis and H. S. Sendov. Nonsmooth analysis of singular values. II. Applications. SetValued Anal., 13(3):243-264, 2005.

[12] A. Rubinov. Abstract convexity and global optimization, volume 44 of Nonconvex Optimization and its Applications. Kluwer Academic Publishers, Dordrecht, 2000.

[13] A. M. Rubinov and Z. Y. Wu. Optimality conditions in global optimization and their applications. Math. Program., 120(1, Ser. B):101-123, 2009.

[14] I. Tsevendorj. Piecewise-convex maximization problems: global optimality conditions. $J$. Global Optim., 21(1):1-14, 2001.

[15] Z. Y. Wu, V. Jeyakumar, and A. M. Rubinov. Sufficient conditions for global optimality of bivalent nonconvex quadratic programs with inequality constraints. J. Optim. Theory Appl., 133(1):123-130, 2007.

[16] Z. Y. Wu and A. M. Rubinov. Global optimality conditions for some classes of optimization problems. J. Optim. Theory Appl., 145(1):164-185, 2010.

Inria, Domaine de Voluceau, Rocquencourt, B.P. 105, 78153 Le Chesnay Cedex, France E-mail address: Dominique.Fortin@inria.fr

Laboratoire PRism, Université de Versailles, 45, aVenue des États-Unis, 78035 VerSailles Cedex, France

E-mail address: Ider.Tseveendorj@prism.uvsq.fr 